\section{Human psychological and sociological factors influencing the suitability of species to be pets}

\section{Martin Whitehead \\ Chipping Norton Veterinary Hospital, Chipping Norton, UK}

\section{OBJECTIVES}

Various schemes have been proposed for assessing the suitability of species to be pets, with the aim of guiding possible future legislation regarding pet keeping and/or allowing animals to be labeled at point of sale with regard to their difficulty-of-keeping and, therefore, risk of poor welfare in captivity. This study aimed to identify factors missing from these schemes.

\section{METHODS}

Critical review of existing schemes.

\section{RESULTS}

Existing schemes are based primarily on properties of the animals themselves, e.g., adult size, potential lifespan, physical or zoonotic risk to humans, how stressful the species may find captivity and human contact, is the animal captive bred or wild caught, and dietary and environmental needs - some of these factors determining the space, equipment, expense and knowledge-base required. Additional factors concern the availability of necessary resources in our society, including appropriate food, equipment, knowledge and informed veterinary care. Each factor varies across species in such a way as to influence the species' difficulty-of-keeping and so risk of poor welfare in captivity. Human factors also vary across species in such a way as to influence the species' welfare as pets, yet most of these important psychological and sociological factors are not included in existing schemes. These factors include human ability to recognise poor welfare in animals, empathy (the ability to perceive another's condition from their perspective), emotional attachment to animals, and the species' moral importance in our society.

\section{STATEMENT}

Existing schemes to assess suitability of species to be pets are incomplete because they do not incorporate relevant psychosocial factors.
Detection of rabbit haemorrhagic disease virus in a veterinary laboratory

\section{Rachel E. Marschang, Karola Weider, Eva-Maria Klas, Hanna Erhard, Claudia Laik-Schandelmaier}

Laboklin GmbH \& Co. KG, Bad Kissingen, Germany

\section{OBJECTIVES}

Rabbit haemorrhagic disease (RHD) is a severe infectious disease of domestic rabbits caused by a calicivirus (RHDV). In recent years, a virus variant, RHDV2 has emerged as an important cause of disease in these animals in Europe and has also been described in other parts of the world. RHD is often a peracute disease with high mortality rates. Vaccination is possible, although vaccination against RHDV1 does not always protect against RHDV2, and new vaccination strategies have been developed. The objective of this study was to determine the prevalence of RHDV1 and 2 in clinical samples in Europe.

\section{METHODS}

The number of submissions of diagnostic samples for the detection of RHDV to a commercial laboratory was monitored for 2015 and 2016 and detection of RHDV1 and RHDV2 was carried out by PCR.

\section{RESULTS}

The number of samples submitted rose significantly from 66 in 2015 to 229 from January to September 2016 RHDV was detected in one third to three fourths of the animals tested in any given month, with only RHDV2 detected in 2015, while sporadic cases of RHDV1 were detected in 2016, with the majority of cases remaining RHDV2 associated. Pathology of affected animals was similar for both virus types, with predominantly hepatic necrosis noted in cases submitted for necropsy.

\section{STATEMENT}

These findings reflect the increased importance of this disease and specifically the new virus variant RHDV2 in pet rabbits, while confirming that RHDV1 remains active in Europe. 